[0212-7199(2002) 19: 9; pp 470-472]
ANALES DE MEDICINA INTERNA Copyright $\odot 2002$ ARAN EDICIONES, S.L.

AN MED INTERNA (Madrid) Vol. 19, N. $^{\circ} 9$, pp. $470-472,2002$

\title{
Camptocormia: una enfermedad muscular infrecuente
}

\author{
Mª M. JIMÉNEZ GONZÁLEZ, M. PONS SERRA*, C. CASTAÑO MORENO*, \\ L. MONÉS JIMÉNEZ**, F. MARTÍNEZ RODENAS*** \\ Medicina Familiar y Comunitaria. *Servicio de Reumatología. **Servicio de Radiología. \\ ***Servicio de Cirugía General. Hospital Municipal de Badalona. Barcelona
}

\author{
CAMPTOCORMIA: A INFREQUENT MUSCULAR DISEASE
}

\begin{abstract}
RESUMEN
La camptocormia es una entidad caracterizada por una cifosis lumbar reductible en decúbito supino, asociada trastornos tomodensitométricos e histológicos en los músculos paravertebrales del segmento lumbar. Aportamos un nuevo caso correspondiente a una mujer de 70 años con dolor lumbar crónico y cifosis dorsolumbar reductible, en quien la tomografia computarizada mostró imágenes compatibles con degeneración grasa y atrofia de la musculatura lumbar, hallazgos que se confirmaron en el estudio histológico.
\end{abstract}

PALABRAS CLAVE: Camptocormia. Cifosis reductible.

\begin{abstract}
The camptocormia is an entity characterized by a kyphosis lumbar reductible in supine decubitus, associated to dysfunctions in computed tomography scans and histologics in the paravertebrals muscles of the lumbar segment. We contribute a new case corresponding to a 70 yearold woman with chronic lumbar pain and kyphosis dorsolumbar reducti ble, in who the on-line tomography showed compatible images with fatty degeneration and atrophy of the lumbar musculature, discoveries that were confirmed in the histologic study.
\end{abstract}

KEY WORDS: Camptocormia. Reductible kyphosis.

Jiménez González MM, Pons Serra M, Castaño Moreno C, Monés Jiménez L, Martínez Rodenas F. Camptocormia: una enfermedad muscular infrecuente. An Med Interna (Madrid) 2002; 19: 470-472.

\section{INTRODUCCIÓN}

El término camptocormia deriva del griego Kamptein (curva) y Kormós (tronco) $(1,2)$. Fue descrita por primera vez por Brodie en 1837 como un trastorno psiquiátrico histérico. Posteriormente se observó en reclutas en la $1^{\mathrm{a}}$ y $2^{\mathrm{a}}$ Guerra Mundial que presentaban dolor y debilidad de la musculatura paravertebral dorso-lumbar $(3,4)$. Hasta inicios de la década de los 90 esta entidad fue considerada de origen psiquiátrico $(3,4)$ y es a partir de entonces, a raíz de diversos estudios (58), cuando pasó a considerarse como una enfermedad con base orgánica. En 1996 Karras (9) planteó la diferenciación entre dos procesos hasta entonces etimológica y clínicamente confundidos: camptocormia y cormoptosis. La cormoptosis se referiría al cuadro de dolor lumbar y cifosis que se presenta en sujetos jóvenes y que tendría una etiología psiquiátrica, mientras que la camptocormia se referería a un proceso que cursaría con afectación de la musculatura paravertebral.

La camptocormia es una enfermedad de afectación muscular poco frecuente, de aparición tardía (5). Su sintomatolo- gía consiste básicamente en lumbalgia crónica asociada a una cifosis secundaria a debilidad de la musculatura paravertebral lumbar. La cifosis se incrementa con la fatiga (6), es reductible completamente en decúbito supino y presenta una relación mujer/hombre de 4/1. En un $20 \%$ de los casos se asocia aun déficit moderado de la musculatura escapular o pelviana. En un $75 \%$ de los casos según Laroche $\mathrm{M}(2,5)$ hay antecedentes familiares, no descritos por otros autores. Su transmisión genética es dominante de penetrancia variable (5).

Para realizar su diagnóstico es preciso que esta clínica se acompañe de ciertos criterios tomodensitométricos e histológicos. En nuestra consulta pudimos valorar el siguiente caso clínico.

\section{CASO APORTADO}

Paciente de 70 años, mujer, que presentaba antecedentes de lumbalgia mecánica de larga evolución. Acudió a la consulta de reumatología por presentar desde hacía más de 15 días dolor lumbar irradiado a pierna izquierda, con empeoramiento en la marcha, dolor en

Trabajo aceptado: 3 de abril de 2001

Correspondencia: Ma. M. Jiménez González. Avda. Santa Coloma, nº 91, 1ª 4. 08922 Sta. Coloma de Gramanet. Barcelona. e-mail: 31297jma@comb.es. 
ambos hombros que empeoraba por la noche y cervicalgia mecánica. No refería síndrome tóxico ni fiebre.

Como antecedentes patológicos presentaba un ingreso por trombosis venosa profunda con tromboembolismo pulmonar asociado hace 7 años, con una pneumonia intrahospitalaria durante el mismo ingreso. Su medicación habitual era Meloxicam $15 \mathrm{mg} 1 \mathrm{compr} / 24$ horas.

Exploración física: buen estado general, normohidratada y normocoloreada. Auscultación cardiorrespiratoria normal. Abdomen blando, sin puntos dolorosos ni visceromegalias. Las maniobras de elongación radicular eran negativas. Los reflejos osteotendinosos estaban presentes y eran simétricos. La fuerza y la sensibilidad de las extremidades superiores e inferiores estaban conservadas. Destacaba cifosis dorso-lumbar en bipedestación, adoptando una posición del tronco (y secundariamente de las extremidades superiores) hacia delante. Presentaba dolor a la presión de la columna dorso-lumbar y a la palpación de la musculatura paravertebral, con marcada debilidad de la musculatura paravertebral y dificultad para realizar la extensión de la columna vertebral lumbar en bipedestación. El resto de la exploración estaba dentro de la normalidad.

Exploraciones complementarias: hemograma, VSG, glicemia, urea, creatinina, ionograma, colesterol, AST, ALT, GGT, fosfatasa alcalina, CK y aldolasa dentro de la normalidad. El HLA B27 fue negativo. Se practicó un electromiograma de la musculatura paravertebral desde L3 hasta L5 donde no se apreció actividad que sugiriese lesión neurógena o miógena inflamatoria.

En el TC lumbar se observó una discopatía severa L4-L5, L5-S1. Las imágenes descritas se acompañaban de atrofia con degeneración grasa de la musculatura paravertebral. Se realizó una biopsia de la musculatura paravertebral lumbar derecha en la que evidenció infiltración adiposa y atrofia de fibras musculares. Estos hallazgos fueron los que sugirieron el diagnósico de camptocormia.

\section{DISCUSIÓN}

El término camptocormia ha sufrido un cambio importante desde el punto de vista etiopatogénico en la última década, pasando tener un origen psiquiátrico a ser una entidad con base orgánica a raíz de estudios realizados en los últimos años.

La camptocormia se podría definir como una afectación de la musculatura paravertebral dorso-lumbar que da lugar a una cifosis adquirida en el adulto que revierte espontáneamente en la posición en decúbito supino, con imposibilidad por parte de los pacientes de realizar una hiperextensión del tronco en bipedestación.

En las exploraciones complementarias observamos que los enzimas musculares están dentro de los límites de la normalidad, la tomografía computarizada está alterada en todos los casos $(1,2,5,7,8,10)$ y en la histología encontramos una capa fibrohialina en el endomisio de las fibras musculares de forma constante y a menudo lobulada, de predominio periférico con un aumento de las fibras musculares tipo $1(1,5)$. Algunos autores (5) evidencian alteraciones mitocondriales en las fibras musculares afectadas. El electromiograma (EMG) es de difícil interpretación por la imposibilidad por parte de los pacientes de realizar una hiperextensión del tronco durante la exploración. En la mayoría de casos el EMG muestra un patrón de miopatía (no neurógena) aunque en nuestro caso no se observaron alteraciones.

El diagnóstico diferencial debemos hacerlo con procesos que presenten afectación dorso-lumbar y atrofia de la musculatura paravertebral.

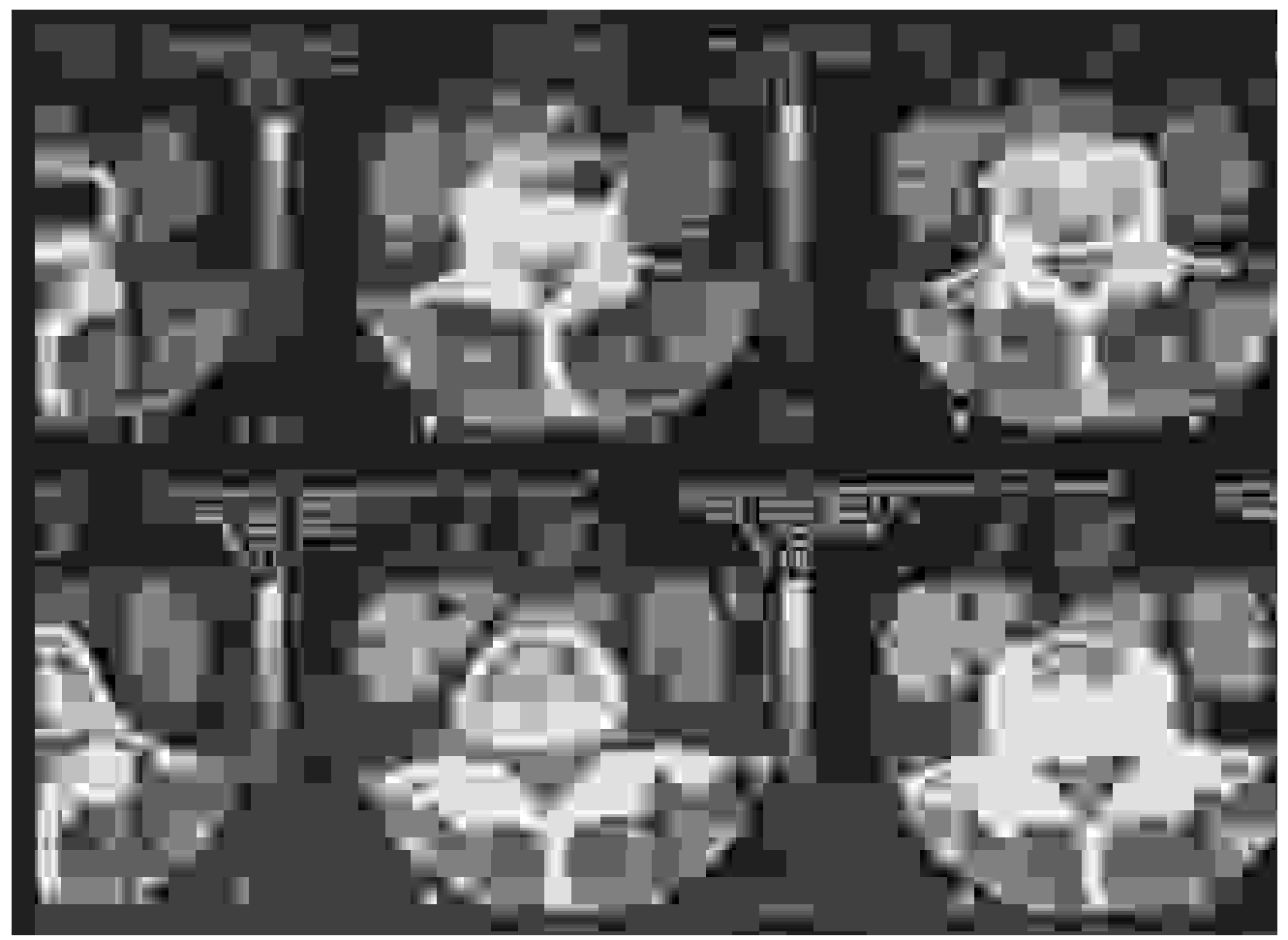

Fig. 1. Corte transversal de la tomografía computarizada (TC) a nivel lumbar con infiltración grasa de la musculatura paravertebral. 
Para el diagnóstico de esta rara entidad se han propuesto 3 grupos de criterios diagnósticos: clínicos, tomodensitométricos y anatomopatológicos (5):

\section{- Criterios clínicos:}

- Proyección anterior del tronco a partir del raquis lumbar con imposibilidad de corrección de éste en bipedestación. La curvatura del raquis es reducible totalmente en decúbito y se agrava con la fatiga.

- Edad superior a 50 años.

- Ausencia de etiología ósea, canal lumbar estrecho, síndrome extrapiramidal, alteración neuromuscular (una afectación moderada de las cinturas escapular o pélvica no es criterio de exclusión), distrofias, osteomalacia o tratamiento con corticoterapia.

-Criterios tomodensitométricos: volumen muscular normal, con hipodensidad e infiltración grasa, heterogenea, de la musculatura paravertebral de predominio distal (D10 a L5).

-Criterios anatomopatológicos: fibrohialinosis endomisial (a menudo lobulada).

En nuestra paciente el diagnóstico se basó en la anamnesis, en la exploración física y en las características de la tomografía computarizada (Figs. 1 y 2).

El tratamiento de esta entidad aún no está bien definido. Basándose en una probable etiología miopática primaria se ha tratado con glucocorticoides (7), siendo eficaz en la mayoría de casos, aunque la dosis y la vía de administración correctas aún no están bien definidas. En nuestro caso se pautó tratamiento analgésico y fisioterápico con buena respuesta clínica y mejoría de su capacidad funcional.

\section{Bibliografía}

1. Delisle MB, Laroche M, Dupont H, Rochaix P, Rumeau JL. Morphological analyses of paraspinal muscles: comparison of progressive lumbar kyphosis (camptocormia) and narrowing of lumbar canal by disc protusions. Neuromuscul Disord 1993; 3: 579-82.

2. Laroche M, Delisle MB, Ariza R, Lagarrigue J, Mazieres B. Is camptocormia a primary muscular disease? Spine 1995; 20: 1011-6.

3. Soreff J. Camptocormia. Arch Orthop Trauma Surg 1983; 101: 151-2.

4. Rosen JC, Frymoyer JW. A review of camptocormia and an unusual case in the female. Spine 1985; 10: 325-7.

5. Laroche M, Delisle MB. La camptocormie primitive est une myopathie para-vertébrale. Rev Rhum (Ed Fr) 1994; 61: 481-4.

6. Laroche M, Delisle MB, Mazières B, Rascol A, et al. Myopathie tardive localisée aux muscle spinaux: une cause de cyphose lombaire acquise de l'adulte. Rev Rhum (Ed Fr) 1991; 58: 829-38.

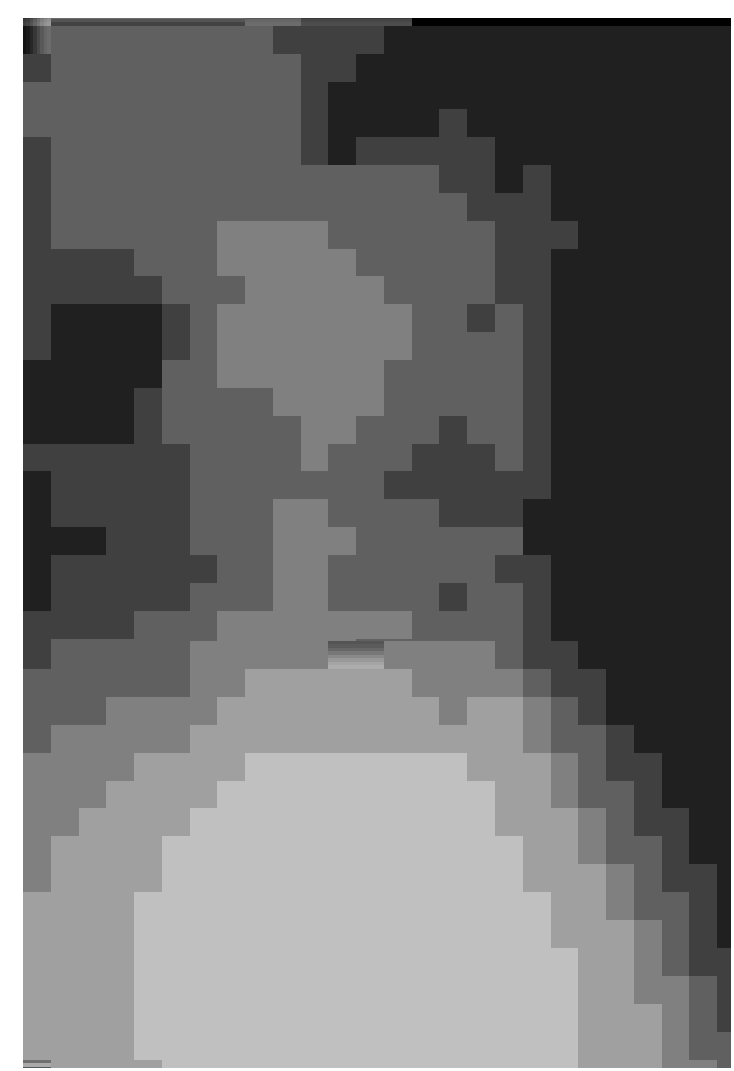

Fig. 2. Corte sagital de la TC de columna lumbar.

7. Hilliquin $P$, Menkès CJ, Laoussadi $S$, Job-Deslandre C, Serratrice G. Camptocormie du sujet âgé. Une nouvelle entité par atteinte des muscles paravertébraux? Rev Rhum Mal Osteoartic 1992; 59: 169-75.

8. Poullin P, Daumen-Legre V, Serratrice G. La camptocormie du sujet agé: myopathie ou dystonie musculaire? Rev Rhum (Ed Fr) 1993; 60: $159-61$.

9. Karras D. Camptocormia or cormoptosis? The etymology of the word. Ann Rheum Dis 1996; 55: 858

10. Laroche M, Rousseau H, Mazières B, Bonafé A, Joffre F, Arlet J. Intérêt de la tomodensitométrie dans la pathologie musculaire. Observations personnelles et revue de la littérature. Rev Rhum (Ed Fr) 1989; 56: 433-9.

11. Morlà RMa $\mathrm{R}^{\mathrm{a}}$, Cayetano A, Ramentol M, Peri J, Casals E, Obach J. Camptocormia (cifosis lumbar reductible): presentación de cinco casos. Rev Esp Reumatol 1999; 26: 282. 